Iranian

Volume 12 Number 3 (June 2020) 249-255

\title{
Immune response in cutaneous leishmaniasis patients with healing vs. non-healing lesions
}

\author{
Akram Miramin-Mohammadi' ${ }^{1}$ Amir Javadi², Seyed Ebrahim Eskandari' ${ }^{1}$ Hossein Mortazavi \\ Mahmoud Nateghi Rostami ${ }^{4}$, Ali Khamesipour ${ }^{1^{*}}$ \\ ${ }^{1}$ Center for Research and Training in Skin Diseases and Leprosy, Tehran University of Medical Sciences, \\ Tehran, Iran \\ ${ }^{2}$ Department of Social Medicines, Qazvin University of Medical Sciences, Qazvin, Iran \\ ${ }^{3}$ Department of Dermatology, Tehran University of Medical Sciences, Razi Hospital, Tehran, Iran \\ ${ }^{4}$ Department of Parasitology, Pasteur Institute of Iran, Tehran, Iran
}

Received: April 2020, Accepted: May 2020

\begin{abstract}
Background and Objectives: The outcome of Leishmania infection mainly depends upon the Leishmania species which causes the disease and the generation of the type of host immune response, the healing process and protection in leishmaniasis depends upon induction of Th1 response. In this study, the Th1/Th2 cytokine profile in cutaneous leishmaniasis (CL) is evaluated.

Materials and Methods: This study was carried out in leishmaniasis clinic of CRTSDL, TUMS, during March 2018 to March 2019. Peripheral blood mononuclear cells (PBMC) of volunteers with active healing and non-healing lesion (s) of cutaneous leishmaniasis (CL), volunteers with and without history of CL were cultured and stimulated with Soluble Leishmania antigen (SLA). The supernatants were collected and the levels of IFN- $\gamma$, IL-5 and IL-10 were titrated using ELISA method.

Results: The results showed a significantly higher levels of IFN- $\gamma$ in volunteers with active CL healing form $(p<0.005)$, history of CL $(p<0.005)$ than healthy volunteers. A significantly $(p<0.005)$ higher level of IFN- $\gamma$ was seen in volunteers with active healing form of lesion than non-healing form. There was a significantly $(p<0.005)$ higher level of IL-10 in volunteers with a history of non-healing form and active non-healing form of CL. There was no significant difference in IL-5 production in PBMC of different groups.

Conclusion: IFN- $\gamma$ production starts at early stage of cutaneous leishmaniasis and enhance during course of lesion healing, IFN- $\gamma$ level is significantly higher in all patients compared to healthy volunteers, IFN- $\gamma$ is significantly higher in patients with healing form than non-healing form of lesion.
\end{abstract}

Keywords: Cutaneous leishmaniasis; Soluble Leishmania antigen (SLA); Immune response; Interferon gamma (IFN $\gamma$ ); Interleukin 10 (IL-10); Interleukin 5 (IL-5)

${ }^{*}$ Corresponding author: Ali Khamesipour, Ph.D, Center for Research and Training in Skin Diseases and Leprosy, Tehran University of Medical Sciences, Tehran, Iran. Tel: +98-21-88970657

Fax: +98-21-88970658

Email: khamesipour@tums.ac.ir

\section{INTRODUCTION}

Leishmaniasis is endemic in 102 countries and is the main health problem in some of the endemic regions which are the poorest areas of the world. Leishmaniasis is endemic in 14 of $22 \mathrm{WHO} / \mathrm{EMRO}$ region countries. Annually, 200,000-400,000 people devel- 